Article

\title{
Extra-Dimensional "Metamaterials": A Model of Inflation Due to a Metric Signature Transition
}

\author{
Igor I. Smolyaninov
}

Electrical and Computer Engineering Department, University of Maryland, College Park, MD 20742, USA; smoly@umd.edu

Received: 2 June 2017; Accepted: 18 September 2017; Published: 20 September 2017

\begin{abstract}
Lattices of topological defects, such as Abrikosov lattices and domain wall lattices, often arise as metastable ground states in higher-dimensional field theoretical models. We demonstrate that such lattice states may be described as extra-dimensional "metamaterials" via higher-dimensional effective medium theory. A $4+1$ dimensional extension of Maxwell electrodynamics with a compactified time-like dimension is considered as an example. It is demonstrated that from the point of view of macroscopic electrodynamics an Abrikosov lattice state in such a $4+1$ dimensional spacetime may be described as a uniaxial hyperbolic medium. Extraordinary photons perceive this medium as a $3+1$ dimensional Minkowski spacetime in which one of the original spatial dimensions plays the role of a new time-like coordinate. Since the metric signature of this effective spacetime depends on the Abrikosov lattice periodicity, the described model may be useful in studying metric signature transitions.
\end{abstract}

Keywords: metamaterial; inflation

\section{Introduction}

Metric signature change events (in which a phase transition occurs between, for example, a $(-,-,+,+)$ and $(-,+,+,+)$ spacetime signature) are being studied in many modified general relativity and quantum gravity theories (see for example [1,2], and the references therein). Studying such events represents an obvious challenge for field theory. In general, it is predicted that a quantum field theory residing on a spacetime undergoing a signature change reacts violently to the imposition of the signature change. Both the total number and the total energy of the particles generated in a signature change event are formally infinite [1,2]. Therefore, such a metric signature transition shares some similarities with the cosmological big bang. Both events lead to the creation of a Minkowski spacetime, and a large number of particles populate this spacetime. Very recently, we described a hyperbolic metamaterial system, which undergoes a lower dimensional version of such a metric signature transition [3]. Hyperbolic metamaterials are artificial uniaxial materials in which the dielectric permittivity has different signs along different orthogonal directions. These metamaterials may be used to model a $2+1$ dimensional Minkowski spacetime in which the role of time is played by one of the spatial coordinates. When a metamaterial is built and illuminated with a coherent extraordinary laser beam, the stationary pattern of light propagation inside the metamaterial may be treated as a collection of particle world lines, which represents a complete "history" of this $2+1$ dimensional Minkowski spacetime [4]. It appears that in a very strong magnetic field, the physical vacuum itself behaves as a hyperbolic metamaterial [5-7].

Motivated by these recent developments, we propose a simple model of a $3+1$ dimensional metric signature change event, which is based on Maxwell electrodynamics residing on a $4+1$ dimensional spacetime with a compactified time-like dimension. While conceptually simple and easy to analyze, our model may be extended to higher-dimensional gauge theories, similar to modern extensions of the 
Kaluza-Klein theory (see for example [8]). As a starting point, we notice that lattices of topological defects, such as Abrikosov lattices [9] and domain wall lattices [10], often arise as metastable ground states both in $4 \mathrm{D}$ and in higher-dimensional field theoretical models. For example, as demonstrated by Chernodub [11], a physical vacuum in a strong magnetic field develops an Abrikosov lattice of superconducting $\rho$ meson condensates. We will demonstrate that such lattice states may be described as extra-dimensional "metamaterials" via higher-dimensional effective medium theory.

\section{Methods}

Higher-dimensional extensions of Maxwell electrodynamics have been described, for example, in Reference [12]. We apply such an extension to a $4+1$ dimensional spacetime with a compactified time-like dimension:

$$
d s^{2}=-d x_{0}^{2}+d x_{1}^{2}+d x_{2}^{2}+d x_{3}^{2}+d x_{4}^{2}=-\frac{T^{2}}{4 \pi^{2}} d \phi^{2}+d x_{1}^{2}+d x_{2}^{2}+d x_{3}^{2}+d x_{4}^{2}
$$

Maxwell's equations in 4 + 1D in differential form are [12]:

$$
\partial_{i} F^{k i}=J^{k}
$$

where $F^{k i}$ is the electromagnetic field tensor, $J^{k}$ is the current, and the Latin letters range is $\mathrm{I}=0, \ldots, 4$. The four-component electric field is $E^{\alpha}=F^{0 \alpha}$, while the six-component magnetic field is $B^{\alpha \beta}=F^{\alpha \beta}$, where the Greek letters range is $\alpha=1, \ldots, 4$. As a result, Maxwell's equations for the field components may be written as:

$$
\begin{gathered}
\partial_{\alpha} E_{\alpha}=\rho \\
e_{\alpha \beta \gamma} \partial_{\alpha} B_{\beta \gamma}=0 \\
\partial_{\alpha} E_{\beta}-\partial_{\beta} E_{\alpha}=-\partial_{0} B_{\alpha \beta} \\
-\partial_{\alpha} B_{\alpha \beta}=\partial_{0} E_{\beta}+J_{\beta}
\end{gathered}
$$

where $e_{\alpha \beta \gamma}$ is the Levi-Civita symbol. Our goal is to introduce an effective medium description of the lattice media, which would be similar to macroscopic electrodynamics. Let us assume the most simple case in which we only need to introduce one additional field $D_{\alpha}$ as an electric field averaged over macroscopic distances (much larger than the lattice periodicity). Thus, in the absence of "external" charges and currents, our "macroscopic" field equations take the form of:

$$
\begin{gathered}
\partial_{\alpha} D_{\alpha}=0 \\
e_{\alpha \beta \gamma} \partial_{\alpha} B_{\beta \gamma}=0 \\
\partial_{\alpha} E_{\beta}-\partial_{\beta} E_{\alpha}=-\partial_{0} B_{\alpha \beta} \\
-\partial_{\alpha} B_{\alpha \beta}=\partial_{0} D_{\beta}
\end{gathered}
$$

These macroscopic field equations must be supplemented by the permittivity tensor $\varepsilon_{\alpha \beta}$ of the lattice medium:

$$
D_{\alpha}=\varepsilon_{\alpha \beta} E_{\beta}
$$

This permittivity tensor is expected to be frequency-dependent, so we will consider solutions of the form $e^{-i \omega_{n} x_{0}}$, where $\omega_{n}=2 \pi n / T$. Thus, $\varepsilon_{\alpha \beta}$ of the lattice medium will be a function of $n$. Due to the 
symmetries of the Abrikosov lattices and the domain wall lattices considered here, $\varepsilon_{\alpha \beta}$ tensors of these effective media are diagonal:

$$
\varepsilon_{\alpha \beta}=\left(\begin{array}{cccc}
\varepsilon_{1} & 0 & 0 & 0 \\
0 & \varepsilon_{1} & 0 & 0 \\
0 & 0 & \varepsilon_{1} & 0 \\
0 & 0 & 0 & \varepsilon_{4}
\end{array}\right)
$$

with different values $\varepsilon_{4}$ in the $x_{4}$ direction, and $\varepsilon_{1}$ in the other three orthogonal directions, respectively. Numerical values of $\varepsilon_{1}$ and $\varepsilon_{4}$ for the typical lattice media will be evaluated later in this paper. We will consider the propagation of "extraordinary waves" in such a medium. For the extraordinary waves $E_{4} \neq 0$, while for the "ordinary waves" $E_{4} \equiv 0$. We will define the extraordinary wave function as $\psi$ $=E_{4}$ so that the ordinary portion of the electromagnetic field does not contribute to $\psi$. Solving the "macroscopic Maxwell's equations" (4) for $\psi=E_{4}$ we obtain:

$$
\varepsilon_{2} \frac{\partial E_{4}}{\partial x_{0}}=-\frac{\partial B_{14}}{\partial x_{1}}-\frac{\partial B_{24}}{\partial x_{2}}-\frac{\partial B_{34}}{\partial x_{3}}
$$

where $B_{\alpha \beta}$ may be found from the third equation in (4). After simple transformations, the wave equation for the extraordinary field may be written as:

$$
-\frac{1}{\varepsilon_{1}} \frac{\partial^{2} E_{4}}{\partial x_{4}^{2}}-\frac{1}{\varepsilon_{4}}\left(\frac{\partial^{2}}{\partial x_{1}^{2}}+\frac{\partial^{2}}{\partial x_{2}^{2}}+\frac{\partial^{2}}{\partial x_{3}^{2}}\right) E_{4}=\omega_{n}^{2} E_{4}
$$

We note that if $\varepsilon_{1}>0$, while $\varepsilon_{4}<0$, this wave equation coincides with the Klein-Gordon equation in $3+1$ dimensional Minkowski spacetime. However, instead of a compactified $x_{0}$ dimension, the role of time in Equation (8) is played by the spatial dimension $x_{4}$. The diagonal terms of $\varepsilon_{\alpha \beta}$ in Equation (6) play the role of new effective metric coefficients in Equation (8).

\section{Results}

Let us calculate the values of $\varepsilon_{1}$ and $\varepsilon_{4}$ as a function of lattice periodicity for the typical lattice media shown in Figure 1. These calculations can be done in simple analytical form for the most important case of low volume concentration $n$ of the topological defects in vacuum. Let us assume that the response of an individual topological defect to an external electric field may be characterized by dielectric permittivity $\varepsilon_{m}$ (its numerical value will be evaluated later in this paper). Following Reference [13], let us evaluate an integral:

$$
\frac{1}{V} \int\left(D_{\alpha}-E_{\alpha}\right) d V \equiv\left\langle D_{\alpha}\right\rangle-\left\langle E_{\alpha}\right\rangle
$$

in which the integrand differs from zero only inside the defects. Therefore, this integral must be proportional to the volume concentration $n$ of the defects. For the domain wall lattice shown in Figure 1a, field components $E_{1}, E_{2}, E_{3}$, and $D_{4}$ must be continuous. These requirements lead to:

$$
\left\langle D_{4}\right\rangle=\left\langle E_{4}\right\rangle\left(1+n\left(1-\frac{1}{\varepsilon_{m}}\right)\right) \text { and }\left\langle D_{1}\right\rangle=\left\langle E_{1}\right\rangle\left(1+n\left(\varepsilon_{m}-1\right)\right)
$$

Thus, in the leading order, the dielectric permittivity tensor components of the domain wall lattice can be estimated as:

$$
\varepsilon_{4} \approx 1 \text { and } \varepsilon_{1} \approx 1+n \varepsilon_{m}
$$

On the other hand, similar consideration of the Abrikosov lattice shown in Figure $1 \mathrm{~b}$ requires $E_{4}$ to be continuous, leading to:

$$
\varepsilon_{1} \approx 1 \text { and } \varepsilon_{4} \approx 1+n \varepsilon_{m}
$$


Equations (11) and (12) demonstrate that even in the small $n$ limit, $\varepsilon_{1}$ and $\varepsilon_{4}$ may have opposite signs if $\varepsilon_{m}$ is large and negative. Let us evaluate the possible values of $\varepsilon_{m}$.

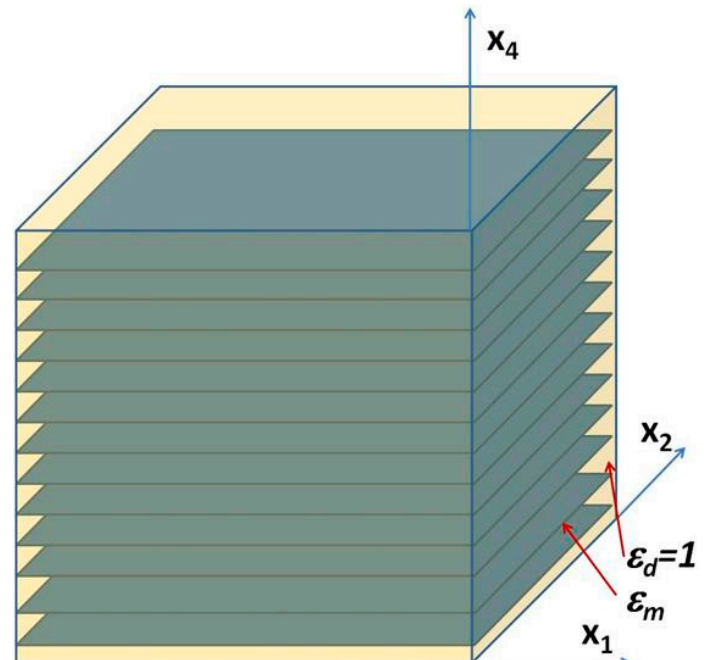

(a)

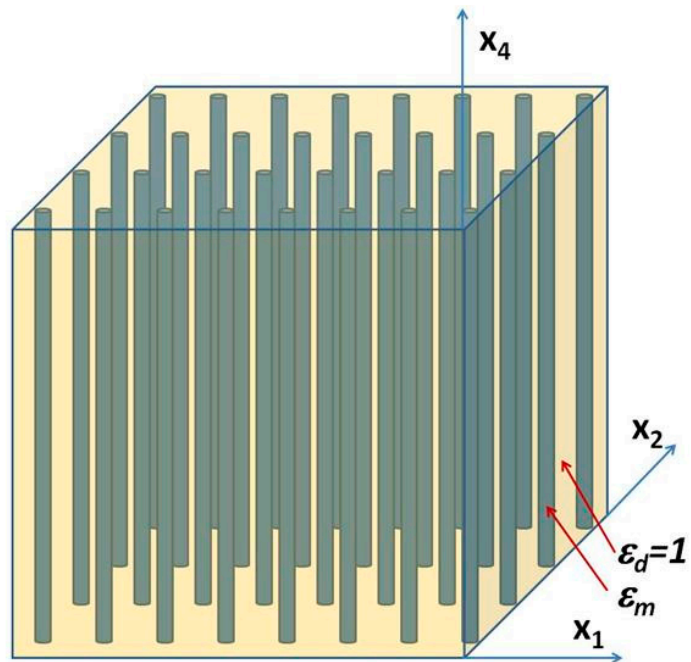

(b)

Figure 1. Lattices of topological defects in a $4+1$ dimensional spacetime considered in our model: (a) Lattice of domain walls; and (b) Abrikosov lattice.

Dissipative behavior, like that in the Ohm law, is inconsistent with the Lorentz symmetry of a physical vacuum. Therefore, from the point of view of the electric conductivity properties, the ground state of the vacuum can either be a superconductor or an insulator [14]. An example of anisotropic superconducting behavior of a physical vacuum in a strong magnetic field due to spontaneous formation of the Abrikosov lattice of $\rho$ meson condensates has been described in Reference [11]. The hyperbolic metamaterial properties of this state have been demonstrated in Reference [5]. The somewhat similar magnetic-field-induced Abrikosov lattice ground state in a $4+1$ dimensional asymptotically Anti-de Sitter space has been found recently by Bu et al. [15] using the holographic approach. Extra-dimensional "holographic" superconductors and inhomogeneous Abrikosov-like states in these superconductors were also considered in much detail in References [16,17]. Within the scope of the holographic model, the frequency-dependent conductivity of the superconducting state can be obtained as (see Equation (4.4) in [16]):

$$
\sigma=\frac{2 a_{2}}{i \omega L_{e f f}^{4} a_{0}}+\frac{i \omega}{2}-i \omega \log L_{e f f}
$$

where $L_{\text {eff }}$ is the effective AdS radius, and $a_{0}$ and $a_{2}$ are the model integration constants. Therefore, in the low frequency limit, the dielectric permittivity $\varepsilon_{m}$ of the holographic superconductor may be obtained as:

$$
\varepsilon_{m}(\omega) \approx \frac{4 \pi \sigma}{i \omega} \approx 2 \pi-4 \pi \log L_{e f f}-\frac{8 \pi a_{2}}{\omega^{2} L_{e f f}^{4} a_{0}} \approx \varepsilon_{\infty}-\frac{\omega_{p}^{2}}{\omega^{2}}
$$

which looks analogous to the conventional Drude model. Thus, $\varepsilon_{m}$ may indeed be large and negative in the low frequency limit, leading to opposite signs of $\varepsilon_{1}$ and $\varepsilon_{4}$ of the Abrikosov lattice. As a result, a $3+1$ dimensional Minkowski spacetime does appear as an effective "macroscopic" metric of the Abrikosov lattice residing in a $4+1$ dimensional spacetime with a compactified time-like dimension. Such a construct, if real, would represent the ultimate manifestation of Mach's principle (see for example [18]) - the spacetime signature of the vacuum would be defined by the distribution of topological defects (matter) in the universe. We should also note that, within the scope of such a 
model, the exact origin of the superconducting behavior (whether its origin is holographic or not) is not important. The only important issue for the validity of this result is that the effective dielectric constant is negative in the superconducting state in the low frequency limit.

Combining Equations (12) and (14) produces the following result for the dielectric permittivity tensor components of the Abrikosov lattice in the low frequency limit:

$$
\varepsilon_{1} \approx 1 \text { and } \varepsilon_{4} \approx 1+n\left(\varepsilon_{\infty}-\frac{\omega_{p}^{2}}{\omega^{2}}\right) \approx 1-n \frac{\omega_{p}^{2}}{\omega^{2}}
$$

where the effective plasma frequency $\omega_{p}$ can be obtained from Equation (14). As can be seen from Equation (8), an effective macroscopic "metric signature change" is observed at the critical value of the Abrikosov lattice density:

$$
n_{c}=\frac{\omega^{2}}{\omega_{p}^{2}}
$$

Typically, the Abrikosov lattice density depends on the magnitude of the external magnetic field $[11,14,15]$. Therefore, an inhomogeneous field distribution may lead to a metric signature transition. However, compared to models described in References [1,2], such a metric signature change does not present a challenge. Calculating the total number and the total energy of particles generated in a signature change event will not lead to divergencies [3]. All the results will be regularized by the finite periodicity of the Abrikosov lattice. These calculations may be performed similar to the method described Reference [3], as follows. The number of photons emitted during the metric signature change transition can be calculated via the dynamical Casimir effect $[19,20]$. The total energy $E$ of emitted photons (per one polarization state) from a metric signature changing volume $V$ depends on the photon dispersion laws $\omega(k)$ before and after the transition (compare to Equation (3) from [20]):

$$
\frac{E}{V}=\int \frac{d^{4} \vec{k}}{(2 \pi)^{4}}\left(\frac{1}{2} \hbar \omega_{1}(k)-\frac{1}{2} \hbar \omega_{2}(k)\right)
$$

where $\omega_{1}(k)$ and $\omega_{2}(k)$ are the photon dispersion relations inside the effective medium before and after the effective "macroscopic metric signature" change. Equation (17) is valid in the "sudden change" approximation, in which the dispersion law is assumed to change instantaneously (the detailed discussion of the validity of this approximation can be found in Reference [20]). Therefore, the number of photons per frequency interval emitted during the transition can be written as:

$$
\frac{d N}{V d \omega}=\frac{1}{2}\left(\frac{d n_{1}}{d \omega}-\frac{d n_{2}}{d \omega}\right)
$$

where $d n_{i} / d \omega$ are the photonic densities of states before and after the transition. At $k>>k_{\max }$, where $k_{\max }$ is the inverse vector of the Abrikosov lattice, the photonic densities of state coincide. Therefore, the integral in Equation (17) remains finite, even though it is quite large, similar to that described in Reference [3], $d N / d \omega \sim k_{\max }{ }^{4}$. In the continuous medium limit in which $k_{\max } \rightarrow \infty$, the number of photons emitted during the transition would diverge in agreement with results obtained in References [1,2].

\section{Discussion}

It is also interesting to note that a particular kind of metric signature transition understood as a macroscopic medium effect may emulate inflation. This fact has been noted in Reference [21] in regard to 3D hyperbolic metamaterials. The metric of a $3+1$ dimensional inflationary de Sitter spacetime may be written as:

$$
d s^{2}=-d x_{4}^{2}+e^{H x_{4}}\left(d x_{1}^{2}+d x_{2}^{2}+d x_{3}^{2}\right)
$$


where the Hubble constant $H \sim \Lambda^{1 / 2}$ ( $\Lambda$ is the cosmological constant). The corresponding Klein-Gordon equation is:

$$
-\frac{\partial^{2} \varphi}{\partial x_{4}{ }^{2}}+\frac{1}{e^{H x_{4}}}\left(\frac{\partial^{2} \varphi}{\partial x_{1}{ }^{2}}+\frac{\partial^{2} \varphi}{\partial x_{2}{ }^{2}}+\frac{\partial^{2} \varphi}{\partial x_{3}{ }^{2}}\right)-H\left(\frac{\partial \varphi}{\partial t}\right)=\frac{m^{2} c^{2}}{\hbar^{2}} \varphi
$$

Now let us extend our $4+1$ dimensional macroscopic electrodynamics model by calculating adiabatic variations of $\varepsilon_{1}$ and $\varepsilon_{4}$ as a function of $x_{4}$. We will demonstrate that the coordinate-dependent diagonal terms of $\varepsilon_{\alpha \beta}$ in Equation (6) play the role of effective metric coefficients, and the resulting effective metric experienced by the extraordinary photons resembles a de Sitter spacetime. Indeed, taking into account spatial derivatives of $\varepsilon_{1}$ and $\varepsilon_{4}$ in Equation (4) results in the following modified equation for $E_{4}$ :

$$
\begin{aligned}
& -\frac{\partial^{2} E_{4}}{\varepsilon_{1} \partial x_{4}^{2}}+\frac{1}{\left(-\varepsilon_{4}\right)}\left(\frac{\partial^{2} E_{4}}{\partial x_{1}^{2}}+\frac{\partial^{2} E_{4}}{\partial x_{2}^{2}}+\frac{\partial^{2} E_{4}}{\partial x_{3}^{2}}\right)+\left(\frac{1}{\varepsilon_{1}^{2}}\left(\frac{\partial \varepsilon_{1}}{\partial x_{4}}\right)-\frac{2}{\varepsilon_{1} \varepsilon_{4}}\left(\frac{\partial \varepsilon_{4}}{\partial x_{4}}\right)\right)\left(\frac{\partial E_{4}}{\partial x_{4}}\right)+ \\
& +\frac{E_{4}}{\varepsilon_{1} \varepsilon_{4}}\left(\frac{1}{\varepsilon_{1}}\left(\frac{\partial \varepsilon_{1}}{\partial x_{4}}\right)\left(\frac{\partial \varepsilon_{4}}{\partial x_{4}}\right)-\left(\frac{\partial^{2} \varepsilon_{4}}{\partial x_{4}{ }^{2}}\right)\right)=\omega_{n}^{2} E_{4}
\end{aligned}
$$

It is easy to see that $\varepsilon_{4}=$ const $>0$ and $\varepsilon_{1} \sim-e^{-H x_{4}}$ (where $x_{4}$ is considered a time-like variable) differs from Equation (20) only by a scaling factor in the limit of a large $H x_{4}$ :

$$
-\frac{\partial^{2} E_{4}}{\partial x_{4}{ }^{2}}+\frac{1}{\varepsilon_{4} e^{H x_{4}}}\left(\frac{\partial^{2} E_{4}}{\partial x_{1}{ }^{2}}+\frac{\partial^{2} E_{4}}{\partial x_{2}{ }^{2}}+\frac{\partial^{2} E_{4}}{\partial x_{3}{ }^{2}}\right)-H\left(\frac{\partial E_{4}}{\partial x_{4}}\right)=-\frac{\omega_{n}{ }^{2}}{e^{H x_{4}}} E_{4} \approx 0
$$

In this limit, Equation (22) describes the propagation of massless particles in the inflationary de Sitter metric described by Equation (19). According to Equation (11), this situation may occur in the lattice of domain walls in which $\varepsilon_{4}=1$, while $\varepsilon_{1}$ may be small and negative near the metric signature transition. In such a case, the spatial distribution of domain walls should be:

$$
n\left(x_{4}\right)=\frac{1+e^{-H x_{4}}}{\left(-\varepsilon_{m}\right)}
$$

near the transition.

We should also note that inflationary scenarios arising due to a metric signature transition have been studied in References [22,23]. However, lattices of topological defects, such as Abrikosov lattices and domain wall lattices, which often arise as metastable ground states in higher-dimensional field theoretical models, have not been previously considered in the context of inflation models. The treatment of such lattices in terms of higher-dimensional "effective media" is a novel feature of our work.

Another potentially relevant body of work is related to the Kibble-Zurek mechanism in cosmology, which predicts domain structure formation in the early universe [24]. This mechanism predicts the density of topological defects in a system which is driven through a continuous phase transition. However, the main novel point of our work is that such topological defect lattices can be simulated by $4+1$ D electromagnetism.

\section{Conclusions}

In conclusion, we have demonstrated that lattices of topological defects, which often arise as metastable ground states in higher-dimensional field theoretical models, may be described as extra-dimensional "metamaterials" via higher-dimensional effective medium theory. A $4+1$ dimensional extension of Maxwell electrodynamics with a compactified time-like dimension was considered as an example. It appears that from the point of view of macroscopic electrodynamics, an Abrikosov lattice state in such a $4+1$ dimensional spacetime may be described as a uniaxial "hyperbolic metamaterial". Extraordinary photons perceive this medium as a $3+1$ dimensional Minkowski spacetime, in which one of the original spatial dimensions (the optical axis of the metamaterial) 
plays the role of a new time-like coordinate. Since the metric signature of this effective spacetime depends on the Abrikosov lattice periodicity, the described model may be useful in studying metric signature transitions. We also note that a particular kind of metric signature transition understood as a macroscopic medium effect may emulate cosmological inflation. Such a situation would represent an ultimate manifestation of Mach's principle (see for example [18]) — the spacetime signature of the vacuum would be defined by the distribution of topological defects (matter) in the universe.

Conflicts of Interest: The author declares no conflict of interest.

\section{References}

1. White, A.; Weinfurtner, S.; Visser, M. Signature change events: A challenge for quantum gravity? Class. Quantum Gravity 2010, 27, 045007. [CrossRef]

2. Dray, T.; Manogue, C.A.; Tucker, R.W. The Scalar field equation in the presence of signature change. Phys. Rev. D 1993, 48, 2587-2590. [CrossRef]

3. Smolyaninov, I.I.; Narimanov, E.E. Metric signature transitions in optical metamaterials. Phys. Rev. Lett. 2010, 105, 067402. [CrossRef] [PubMed]

4. Smolyaninov, I.I.; Hung, Y.J. Modeling of time with metamaterials. J. Opt. Soc. Am. B 2011, 28, 1591-1595. [CrossRef]

5. Smolyaninov, I.I. Vacuum in strong magnetic field as a hyperbolic metamaterial. Phys. Rev. Lett. 2011, 107, 253903. [CrossRef] [PubMed]

6. Smolyaninov, I.I. Planck-scale physics of vacuum in a strong magnetic field. Phys. Rev. D 2012, 85, 114013. [CrossRef]

7. Smolyaninov, I.I. Quantum electromagnetic "black holes" in a strong magnetic field. J. Phys. G Nucl. Part. Phys. 2013, 40, 015005. [CrossRef]

8. Smolyaninov, I.I. Fractal extra dimension in Kaluza-Klein theory. Phys. Rev. D 2002, 65, 047503. [CrossRef]

9. Abrikosov, A.A. Magnetic properties of superconductors of the second group. Sov. Phys. JETP 1957, 5, 1174.

10. Pogosian, L.; Vachaspati, T. Domain wall lattices. Phys. Rev. D 2003, 67, 065012. [CrossRef]

11. Chernodub, M.N. Spontaneous electromagnetic superconductivity of vacuum in a strong magnetic field: Evidence from the Nambu-Jona-Lasinio model. Phys. Rev. Lett. 2011, 106, 142003. [CrossRef] [PubMed]

12. McDavid, A.W.; McMullen, C.D. Generalizing cross products and Maxwell's equations to universal extra dimensions. arXiv 2006, arXiv:hep-ph/0609260.

13. Landau, L.D.; Lifshitz, E.M.; Pitaevskii, L.P. Course of Theoretical Physics; Butterworth-Heinemann: Oxford, UK, 1984; Volume 8.

14. Chernodub, M.N.; Van Doorsselaere, J.; Verschelde, H. Magnetic-field-induced superconductivity and superfluidity of $\mathrm{W}$ and $\mathrm{Z}$ bosons: In tandem transport and kaleidoscopic vortex states. arXiv 2013, arXiv:1203.5963 [hep-ph].

15. Bu, Y.-Y.; Erdmenger, J.; Shock, J.P.; Strydom, M. Magnetic field induced lattice ground states from holography. arXiv 2013, arXiv:1210.6669 [hep-th]. [CrossRef]

16. Siani, M. Holographic superconductors and higher curvature corrections. J. High Energy Phys. 2010, $2010,035$. [CrossRef]

17. Cots, O.D. Composites from Holography and Beyond. Ph.D. Thesis, University of Barcelona, Barcelona, Spain, 2012.

18. Bondi, H.; Samuel, J. The Lense-Thirring effect and Mach's principle. Phys. Lett. A 1997, 228, 121-126. [CrossRef]

19. Schwinger, J. Casimir light: The source. Proc. Natl. Acad. Sci. USA 1993, 90, 2105-2106. [CrossRef] [PubMed]

20. Liberati, S.; Visser, M.; Belgiorno, F.; Sciama, D. Sonoluminescence as a QED vacuum effect. Phys. Rev. D 2000, 61, 085023. [CrossRef]

21. Smolyaninov, I.I.; Hung, Y.J.; Hwang, E. Experimental modeling of cosmological inflation with metamaterials. Phys. Lett. A 2012, 376, 2575-2579. [CrossRef]

22. Ghaffarnejad, H. Wave function of the Universe, preferred reference frame effects and metric signature transition. J. Phys. Conf. Ser. 2015, 633, 012020. [CrossRef] 
23. Ghaffarnejad, H. Brans-Dicke classical cosmology in Einstein frame and metric signature transition. Iranian J. Phys. Res. 2013, 13, 203-211.

24. Kibble, T.W.B. Topology of cosmic domains and strings. J. Phys. A Math. Gen. 1976, 9, 1387. [CrossRef]

(c) (1)

BY 